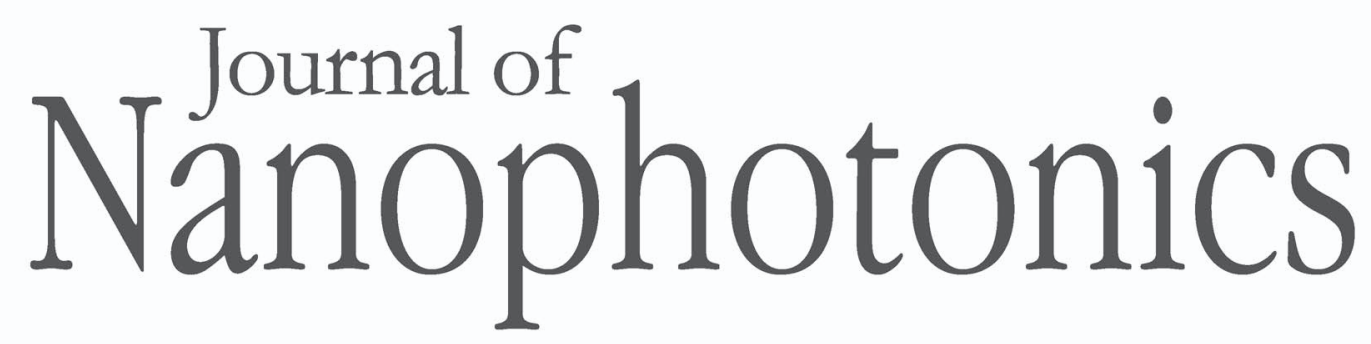

SPIEDigitalLibrary.org/jnp

\title{
Commentary: Nanomanufacturing
}

Tarun Gupta 


\title{
Commentary: Nanomanufacturing
}

\author{
Tarun Gupta \\ Western Michigan University, Department of Industrial and Manufacturing Engineering, \\ Kalamazoo, MI 49008-5336, USA \\ tarun.gupta@wmich.edu
}

Paper 11081CTR received Aug. 2, 2011; revised manuscript received Aug. 12, 2011; accepted for publication Aug. 15, 2011; published online Sep. 16, 2011. [DOI: 10.1117/1.3633245]

Whereas nanotechnology is the study of the properties of matter on the nanoscale, nanomanufacturing is the process of assembling complex structures and products with nanoscale precision and reproducibility. Molecular nanotechnology ${ }^{1}$ requires even more precision: the idea is to precisely place every atom within a product so that components are exactly the same size, of the same type, and comprise the same number and types of atoms.

Nanomanufacturing processes can be classified into three groups when it comes to implementation: i. top-down fabrication, ii. bottom-up assembly, and iii. hybrid methodology. Top-down fabrication requires downscaling existing manufacturing processes. Bottom-up assembly and hybrid methodology require much creativity, particularly to produce similarly dimensioned finished products. The future of nanomanufacturing also depends on the ability to measure both physical and chemical properties at the nanoscale, and therefore on both metrological devices and modeling tools.

Nanomanufacturing business activities can be broadly categorized into three types: i. scaledup production of nanomaterials such as carbon nanotubes, nanowires, and quantum dots; ii. production of tangible goods using nanomaterials; and iii. production of one-of-a-kind nanosystems. Like any other form of manufacturing, nanomanufacturing begins with the concept of a product and market analysis to project business volume, and goes through the following four steps: drafting and design, prototype development, tools development, and industrialization. The last step involves acquisition of capital and equipment, selection and optimization of processes, specifications of raw materials, selection of vendors, management of actual production operation, measurement and quality assurance, and warranty claims leading to changes of design and/or processes which requires the entire sequence of activities to be under constant monitoring and control.

As depicted in Fig. 1, the manufacturing cycle begins with the conceptualization and design of a product. To perform this task effectively and to keep cost per launch down with rapid delivery to the market, reconfigurable computing tools are needed. That means a new class of computeraided design (CAD) tools have to be developed for product concept, drafting and design, and visualization and analysis. CAD tools for nanomanufacturing must be flexible enough to incorporate emerging components, products, and technologies such as silicon nanowires, biofluidic microchips, and DNA self-assembly. Moreover, it is not so much the technology that is important, but it is the massive number of nanoscale devices that would require revolution in a CAD system design to provide the needed functionalities. Due to the sheer number of devices in any nanosystem, the defect rate at the system level is expected to be high. The very small size of an average feature would also potentially contribute to design considerations.

Over the last 10 years the CAD community has been grappling with design tools for nanoscale structures, products and systems. The industry leader, Nanorex Inc., ${ }^{2}$ is actively engaged in developing a comprehensive array of design tools for nanoscale structures in partnership with the academic research community. The company has developed NanoEngineer-1, an open source software for visualization, modeling, and manipulation of DNA, and to make it a framework

$1934-2608 / 2011 / \$ 25.00$ @ 2011 SPIE 


Concept $\rightarrow$ Design $\rightarrow$ Analyze $\rightarrow$ Prototype $\rightarrow$ Tooling $\rightarrow$ Trial Run \& Production

Fig. 1 A typical manufacturing cycle.

that can support and integrate with other computational tools developed by the structural DNA nanotechnology community. ${ }^{3-6}$

Much of the excitement in nanomanufacturing research comes from the creativity required by any bottom-up process. Researchers are trying to design and develop nanoscale features into similar dimensioned finished products by drawing analogies from an abundantly available biological process - self-assembly — which has always been a bottom-up process. However, a more likely scenario would be to fabricate individual features with the top-down approach followed by, or concurrent, assembly using bottom-up approach. Hybrid nanomanufacturing processes are already making headlines with remarkable capabilities. ${ }^{78}$ For example, dip pen technology is relatively inexpensive for laying numerous functional devices on a single sheet of substrate. Near-field optical lithography starts with a wafer sprinkled with carbon nanotubes (CNTs) on the circuit and then e-beams the circuit where the tubes are. The current CNT fabrication processes can produce nanotubes in lengths that could vary up to $300 \%$ of the expected mean length. ${ }^{9}$ The process is found feasible for constrained features under $20 \mathrm{~nm}$; as long as the CNTs length variation within the current capability is not an issue.

In an effort to produce advanced materials using CNTs for specific commercial applications, a single stage nanolithographic process under a controlled environment has been developed. Using this process commercial grade scaled-up production of $6 \mathrm{ft} . \times 8 \mathrm{ft}$. sheets in bundles out of fiber and polycarbonate/polymer for radar evading aircrafts and other equipment has been successfully demonstrated.

Before scaling up any production process we must have metrological equipment in place, since we cannot produce if we cannot measure with needed precision. Measurements are critical for quality assurance in any form of manufacturing, but even more so when the product characteristics at nanoscale are of concern. A key enabling technology for nanomanufacturing is nanometrology. Scanned probe microscopy is essential in nanometrology. Nanoscale measurement problems have been worked upon persistently. From a knowledge of the landscape of nanomanufacturing, measurements of $25-\mathrm{nm}$ features at the rate of $2 \times 10^{10} \mathrm{pixel} / \mathrm{s}$ is projected for 2015 for lithographic processes. ${ }^{10}$ For letter press operation, the technology that is under development is expected to deal with $40-\mu \mathrm{m}$ print press features spread over a sheet and be able to measure feature packs at a rate of $30 \mathrm{~m}^{2} / \mathrm{s}$. As of this writing, technological developments are on track for these targets. Multiple low-power laser beams, each with tractor light effect for trapping independently suspended nanoscale objects in their pathway, are being developed for nanoscale measurements.

The bottom line is that nanomanufacturing is all about "small, light, and cheap," with levels of functional characteristics at times several orders in magnitude higher in performance.

\section{References}

1. E. K. Drexler, Engines of Creation: The Coming Era of Nanotechnology, Anchor Books, New York (1981).

2. E. K. Drexler, http://www.nanoengineer-1.com/content/\#open_source_link.

3. R. Goering, http://www.eetimes.com/electronics-news/4056661/Design-tools-eyenanoscale-realm?pageNumber $=0$ (2005).

4. E. Kim and G. M. Whitesides, "The use of minimal free energy and self-assembly to form shapes," Chem. Mater. 7, 1257-126 (1995).

5. G. M. Whitesides, "Self assembly and nanotechnology," Fourth Foresight Conference on Molecular Nanotechnology (2002). 
6. R. Jackman, J. Wilbur, and G. M. Whitesides, "Fabrication of submicron features on curved surfaces by microcontact printing," Science 269, 664-666 (1995).

7. E. Kim, Y. Xia, and G. M. Whitesides, "Making polymeric microstuctures: Capillary micromolding," Nature 376, 581-584 (1995).

8. A. Kumar, N. A. Abbott, E. Kim, H. A. Biebuyck, and G. M. Whitesides, "Patterned SAMs and meso-scale phenomena," Acc. Chem. Res. 28, 219-226 (1995).

9. K. G. Lee, R. Wi, M. Imran, T. J. Park, J. Lee, S. Y. Lee, and D. H. Kim, "Functionalization effects of single-walled carbon nanotubes as templates for the synthesis of silica nanorods and study of growing mechanism of silica," ACS Nano 4(7), 3933-3942 (2010).

10. L. J. Alexander, "Measurement challenges in nanomanufacturing," Nanofabrication Research Group, Center for Nanoscale Science \& Technology, New England Nanomanufacturing Summit (2010). 